\title{
Towards Maximal Cell Density Predictions for Polymeric Foams
}

\author{
Yeongyoon Kim \\ Department of Physics and Astronomy, University of Waterloo, 200 University Avenue \\ West, Waterloo, Ontario, Canada N2L $3 G 1$ \\ Chul B. Park \\ Microcellular Plastics Manufacturing Laboratory, Department of Mechanical and Industrial \\ Engineering, University of Toronto, 5 King's College Road, Toronto, Ontario, Canada M5S \\ $3 G 8$ \\ P. Chen \\ Department of Chemical Engineering, University of Waterloo, 200 University Avenue West, \\ Waterloo, Ontario, Canada N2L $3 G 1$ \\ Waterloo Institute for Nanotechnology, University of Waterloo, 200 University Avenue \\ West, Waterloo, Ontario, Canada N2L $3 G 1$ \\ Russell B. Thompson \\ Department of Physics and Astronomy, University of Waterloo, 200 University Avenue \\ West, Waterloo, Ontario, Canada N2L 3G1 \\ Waterloo Institute for Nanotechnology, University of Waterloo, 200 University Avenue \\ West, Waterloo, Ontario, Canada N2L 3G1
}

\begin{abstract}
Self-consistent field theory is used to make direct predictions for the maximum possible cell densities for model polymer foam systems without recourse to classical nucleation theory or activation barrier kinetic arguments. Maximum possible cell density predictions are also made subject to constraining the systems to have maximal possible internal interface and to have well formed bubbles (no deviation from bulk conditions on the interior of the bubble). This last condition is found to be the most restrictive on possible cell densities. Comparison is made with classical nucleation theory and it is found that the surface tension is not an important independent consideration for predicting conditions consistent with high cell density polymeric foams or achieving the smallest possible bubble sizes. Instead, the volume free energy density, often labelled as a pressure difference, is the dominant factor for both cell densities and cell sizes.
\end{abstract}

Email address: thompson@uwaterloo.ca (Russell B. Thompson) 


\section{Introduction}

Polymeric foams are made by generating bubbles with a blowing agent of gas or supercritical fluid in a polymer melt and then solidifying the polymer to trap the bubbles [1]. Depending on the material application, different types of foams can be created. For many existing or potential applications, very high quality foams are needed, with the definition of "high quality" also depending on the application. One common ambition is to create foams with very high cell densities, that is, with large numbers of bubbles per unit polymer volume. For this, one wants not only very small bubbles, but also that these cells are positioned close together. The hope is that this will create a large total amount of bubble surface within the polymer matrix and give the foam very good properties. For the same reason, one typically wants well-defined bubbles, in that the cells strongly exclude the matrix polymer. Taken together, a foam with these aspects might be described as a high quality foam.

In order to create the "best" possible foam with given chemistry of polymer and blowing agent, one may have to try a huge number of variations of experimental conditions (temperature, pressure, methods of processing, additives, and so forth). It is desirable to have theoretical guidance that would point to conditions that are more likely to allow the creation of a high quality foam. The most common theoretical methods are based on variations of classical nucleation theory (CNT). CNT has many advantages, most important among them being the simplicity of the approach, but CNT has been shown to be insufficient in many situations $[2,3,4,5,6,7,8,9,10,11]$. There is evidence that it can be used for some polymer foam predictions $[12,13,14,15]$ but for very high quality foams, specifically nano-cellular foams, we have shown in our previous publication that CNT fails badly [16]. Specifically, we showed that when the curvature of the bubble is on the same size scale as the polymer molecules, CNT is no longer an appropriate approach for predicting bubble nucleation rates. It is through such nucleation rates that cell densities would be predicted and so, for high cell density polymeric foams, CNT should make quantitatively wrong, and as we show below, qualitatively incorrect predictions of cell densities.

In this paper, we show that self-consistent field theory (SCFT) provides an in-principle method of predicting cell densities of polymer foams. Unlike CNT, no nucleation energy barrier or nucleation rate need be calculated. Therefore, arguments about exponential pre-factors are rendered irrelevant. Also, no integration over time of the nucleation rate is needed; rather the cell density prediction is a direct result of the SCFT calculation. Indeed, using SCFT, one can find cell densities corresponding to the bubble critical radius, the radius of maximal bubble surface area and the radius of maximal polymer exclusion from the interior of the bubble (more will be said about these conditions in the Results and Discussion section). Thus one can choose what is meant by the "best" possible polymeric foam and select the corresponding cell density for that case. Since SCFT is an equilibrium statistical mechanical approach, and 
therefore includes no kinetic information, the best methods for processing the polymer foam are not addressed by SCFT. Rather, SCFT provides an upper bound on the cell density, with experiment expected to find lower values of cell density. If one is interested in nanocellular foams for example, one can use SCFT to avoid spending too much time experimentally searching points of the parameter space for which SCFT maximal possible cell density predictions are low and concentrate on areas where the cell density has the possibility of being high.

We have given in depth presentations of the SCFT method in previous publications $[16,17,18,19]$ and there are many excellent reviews [20, 21, 22]. The SCFT theory is therefore only briefly reviewed here in section 2. As a first attempt at cell density predictions, we will follow the formalism of our previous work [16] and limit ourselves to an incompressible equation of state. By this, we mean the system volume doesn't change, regardless of temperature or pressure, so that there is no swelling of the polymer by the gas. This approach has been shown to be sufficient for qualitative understanding [15, 18, 17]. For quantitatively accurate predictions, more realistic equations of state should be used. Incompressible results can then be used as a baseline to isolate equation of state effects. In section 3 we will give some examples of cell density predictions and how we define the "best" possible foam. We will also show qualitative differences in predictions between SCFT and CNT. The origins of the failure of CNT are discussed in depth in our previous work [16]. In section 4 we summarize our results. In particular we find that the volume free energy density (pressure difference) parameter must be considered the controlling factor for cell density predictions, rather than the surface tension.

\section{Theory}

Self-consistent field theory (SCFT) is a mean field, equilibrium statistical mechanical methodology. It neglects fluctuations and, in the form presented here, does not deal with dynamics. It most commonly uses a Gaussian string model to represent polymeric degrees of freedom. Derivational details and possible modifications and extensions have been reviewed in several works [20, 21, 22]. The SCFT model for a bubble of fluid in a polymer matrix can be summarized by the free energy functional [16]

$$
\begin{aligned}
\tilde{\mathcal{F}} \equiv \frac{N F}{\rho_{0} k_{B} T V}= & -\left(1-\phi_{s}\right) \ln \left[\frac{Q_{p}}{V\left(1-\phi_{s}\right)}\right]-\frac{\phi_{s}}{\alpha} \ln \left(\frac{Q_{s} \alpha}{V \phi_{s}}\right) \\
& +\frac{1}{V} \int d \mathbf{r}\left[\chi N \varphi_{p}(\mathbf{r}) \varphi_{s}(\mathbf{r})-w_{s}(\mathbf{r}) \varphi_{s}(\mathbf{r})-w_{p}(\mathbf{r}) \varphi_{p}(\mathbf{r})\right](1)
\end{aligned}
$$

where $\varphi_{p}(\mathbf{r})$ and $\varphi_{s}(\mathbf{r})$ are the local (position dependent) volume fractions of polymer and gas (or fluid), respectively. The subscript " $s$ " is used for the gas volume fraction since it is customary in SCFT to refer to molecules lacking polymeric internal degrees of freedom as "solvent" molecules. We will continue to use this term in this paper. The total system volume fractions for polymer 
and solvent are $\left(1-\phi_{s}\right)$ and $\phi_{s}$, respectively, and single molecule partition functions are given by $Q_{p}$ and $Q_{s}$, respectively. The ratio of the volume of a solvent molecule to a polymer molecule is denoted by $\alpha$, with the volume of one polymer segment being $\rho_{0}^{-1}$ and the degree of polymerization being $N$. The segregation between solvent molecules and polymer segments is given by a FloryHuggins parameter $\chi$. This parameter is inversely proportional to temperature $T$ and is also related to the chemistry of the polymer and solvent molecules. The mean fields felt by each solvent molecule or polymer segment due to interactions with all other molecules and segments in the system are given by $w(\mathbf{r})_{s}$ or $p$. The left hand side of $(1)$ is the system free energy $(F)$ per system volume $(V)$, made dimensionless using appropriate factors. In this work, all lengths are phrased in terms of the radius of gyration of a polymer, $R_{g}$. Variation of equation (1) with respect to all functions yields a set of coupled, non-linear equations to be solved self-consistently. In order to complete this set, one also needs the equation of state. In this work we follow our previous derivation and, for simplicity, choose an incompressible equation of state [16]. This is given by the equation

$$
\varphi_{p}(\mathbf{r})+\varphi_{s}(\mathbf{r})=1 .
$$

The SCFT equations are solved numerically as described in previous work $[16$, $17,18,19]$ and results have been found to be in good qualitative agreement with polymer surface tension experiments $[23,24,25,26,19,27,28]$.

In this formalism, the overall solvent (gas) volume fraction $\phi_{s}$ is held constant at some experimental value and one changes the size of the bubble by changing the size of the system volume $V$. The volume $V$ is therefore the average volume per bubble and is the size of the calculational box in SCFT. Therefore, as new generations of bubbles nucleate and grow, the single, typical, bubble that we study incorporates the depletion of dissolved gas in the system. This is in the same spirit as the approach of Amon and Denson [29] except that they did not include explicit polymeric degrees of freedom. The typical cell number density would then be trivially found as the inverse of $V$. It is customary in polymer foaming however to define the cell density as the number of bubbles per unit volume of polymer [30]. We follow this convention here and therefore the cell density is the inverse of the product of the calculational box volume times the polymer volume fraction. This would be the best possible cell density to the extent that the extreme case of the kinetic foaming phenomena can be predicted by thermodynamic theory. In reality, the polydispersity of bubble size and kinetic limitations would prevent the experimental system from reaching this cell number density. Nonetheless, the maximal possible cell density still provides the essential guidance of which conditions are favorable for the production of high quality foams and which are not. If the radius of a bubble is defined as the equimolecular surface, as we have done in our previous paper [16] and as we will do here, one can find the cell density as a function of bubble radius. The best possible foam for given input experimental conditions (chemistry or temperature $\chi$, dissolved gas $\phi_{s}$, polymer molecular weight $N$, and so forth) is that foam for which the cell density is highest subject to other relevant constraints. Other considerations might include the smallest possible radius of bubble, the 
maximum amount of bubble surface area per volume of foam, or the smallest radius cell that completely excludes polymer from its interior. We shall examine all these cases in the Results and Discussion section.

This prescription for using SCFT as a guide for predicting good quality foams can be compared and contrasted with CNT and more involved theories based on CNT. Although CNT itself is widely known to have problems, many improved theories are modifications of $\mathrm{CNT}$ and, as such, may point in wrong directions for some applications despite improving CNT quantitatively for others. We have discussed the origins of the failure of CNT in our previous publication [16]. Here, we will look rather at the operational details of CNT predictions, specifically, where CNT can be trusted and where not, based on a comparison with SCFT results to follow in the Results and Discussion section.

The quality of a foam is typically predicted from CNT based on nucleation rates. The nucleation rate is given by

$$
J=J_{0} \exp \left(-\frac{\Delta F^{*}}{k_{B} T}\right)
$$

where $J_{0}$ is a prefactor associated with the characteristic time scales of motion in the system, $k_{B}$ is Boltzmann's constant and $T$ is the temperature [16, 1, 4]. The activation barrier $\Delta F^{*}$ is given by $\mathrm{CNT}$ as

$$
\frac{\Delta F^{*}}{k_{B} T}=\frac{16 \pi}{3}\left(\frac{\gamma^{3}}{\Delta \mathcal{F}_{V}^{2}}\right)
$$

where $\gamma$ and $\Delta \mathcal{F}_{V}$ are the dimensionless surface tension and dimensionless volume free energy density, respectively [31], and are, for CNT, inputs of the theory that are independent of bubble radius $R$. The bubble size in CNT is given by a critical radius (here made dimensionless by dividing by the unit of length $R_{g}$ )

$$
\frac{R^{*}}{R_{g}}=\frac{2 \gamma}{\Delta \mathcal{F}_{V}}
$$

where $\Delta \mathcal{F}_{V}$ is defined to be a positive quantity. Given the inputs $\gamma$ and $\Delta \mathcal{F}_{V}$, the typical bubble size is predicted using (5) and cell densities are found by integrating the nucleation rate expression (3) over time based on the activation barrier (4). One also has to derive through other means a form of the exponential prefactor of (3). CNT can only predict cell densities at the critical radius and gives no information about cell densities at the radius that maximizes the bubble surface area to volume ratio or at the radius at which bubbles size is minimumized without allowing polymer to enter the interior of bubbles - more will be said about these cases in the Results and Discussion section. The parameters $\gamma$ and $\Delta \mathcal{F}_{V}$ are inputs for CNT, found through other theoretical methods or from experiment, but they are outputs of SCFT [16]. We shall see in the Results and Discussion section that the CNT assumption that $\gamma$ and $\Delta \mathcal{F}_{V}$ are not functions of bubble radius will cause qualitative disagreements between CNT and SCFT with respect to predicting under what conditions a high cell density can be created. 


\section{Results and Discussion}

As mentioned, the "best foam" can be defined in several different ways. One can choose the foam with the smallest possible bubbles, that is, with a radius equal to the critical radius. Alternatively, one can choose the foam with the highest amount of internal surface area. Surprisingly, this is, in general, not the same as the foam with the smallest, critical radius, bubbles, as we will show. Lastly, one can choose the foam with significant exclusion of polymer. Again, we will show that this is different from and, in general, more restrictive, than the previous two cases. In other words, both at the critical radius and the radius of maximal bubble surface area, we usually find significant deviations from bulk conditions within the bubbles. In practice, the definition of the "best" foam would depend on the application and would likely be a combination of the above three possibilities. In this paper, we consider all three cases and find that the typical bubble radius is different in these situations. Specifically, we examined systems with $\chi N=160$ and different global volume fraction values in the nucleation and growth region ranging from 0.17 to 0.29 . We calculated the bubble number density, the total bubble surface area per polymer volume, and the solvent volume fraction value at the center of a bubble as a function of bubble radius for each case. As mentioned, in most cases, requiring the bubble to exclude all polymer from the bubble center is the most limiting definition and fixes the bubble size and cell density.

To begin, the simplest attitude, and the one most relevant to the formation of nanocellular foams, is to consider that the smallest possible bubble is the most desirable outcome. This smallest bubble is, of course, just the critical radius. Since in this SCFT methodology, the volume associated with each bubble is adjusted to maintain the correct overall solvent volume fraction, this means that, unlike CNT, we automatically know the average cell number density of the system once the radius has been found. It is just the inverse of the product of the SCFT outputted volume times the polymer volume fraction. The bubble cell density for a variety of solvent volume fractions at a segregation of $\chi N=160$ is shown in figure 1(a). The bubble cell densities at the corresponding critical radii are shown in figure 1(b). It is not surprising that for all cases, the maximum cell density corresponds to the critical radius, that is, the smallest possible bubble. However, although for some values of $\phi_{s}$ the critical radii are similar, for example $\phi_{s}=0.29$ and $\phi_{s}=0.26$, the maximum cell densities can be radically different. Clearly, a prediction of the smallest possible bubble size alone under given conditions is not sufficient to predict the "quality" of a foam. The prediction of higher cell densities with increased blowing agent is consistent with the observations of Goel and Beckman [13] and later observations, for example, references [15, 32, 33, 34].

Surprisingly, the critical radius does not correspond to the maximal bubble surface area per polymer volume. A foam with a small bubble size but very few bubbles will likely not be of much use. It is the very large amount of internal interface that gives foams many of their desirable properties. The SCFT approach allows us to predict the average bubble area per polymer volume which 


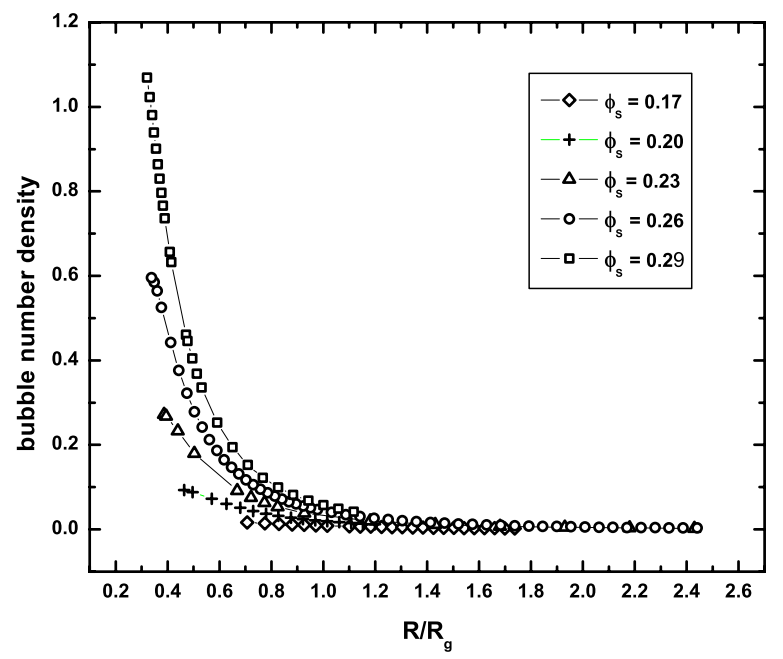

(a)

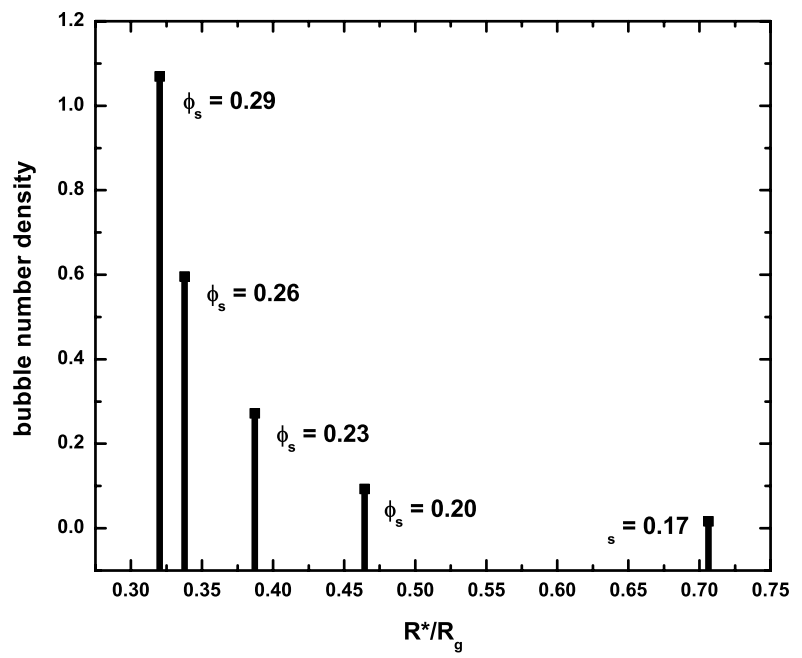

(b)

Figure 1: At $\chi N=160$. (a) Dimensionless bubble number density versus radius of a bubble. (b) Dimensionless bubble number density at the critical radius of a bubble at different solvent density systems. 
is shown in figure 2(a) for $\chi N=160$ and a selection of $\phi_{s}$ values. One observes that the maximum bubble surface area per polymer volume, $A / V_{p}$, does not occur at the critical radius, but at slightly larger radii. In figure 2(b), the cell density corresponding to this maximum $A / V_{p}$ is shown. From figure 1 (a) one also notices that there are fewer bubbles at the maximum $A / V_{p}$ radius than at the critical radius. Therefore the increased area per polymer volume is not arising due to merely having more bubbles. Instead, SCFT is showing us that initially, bubbles harvest gas through a process of re-arrangement of molecules in their own localities with less interaction with other bubbles. In this way, they increase their radii and area while changing their associated volume more slowly. After the $A / V_{p}$ maximum radius however, the bubbles must compete more with each other for gas. They harvest gas molecules from other bubbles and their associated volumes must grow more quickly relative to their surface areas. From both figures 1 and 2, it is clear that the "best" foam is one with a high gas content. This gives many cells with large total surface area. In practice however, too much fluid brings one close to the spinodal,that is, the limit of metastability of the mixture, where nucleation and growth ceases and well formed spherical bubbles will not be created. Rather, one would expect to get bicontinuous structures. One needs to keep the gas content low enough to avoid the spinodal but high enough to achieve large cell densities and plentiful bubble surface area. SCFT, in principle, offers a method to predict both these quantities for given conditions and gas content [35].

While the requirement for a maximal bubble area per polymer volume increases the radius of bubbles beyond the critical radius, so too does a requirement that the bubbles be, individually, of "good quality". By this, we mean that the interior of the bubble should be well segregated and almost free of polymer, that is, the interior of the bubbles should reach bulk conditions. Poorly formed bubbles would, for most applications, undermine the usefulness of the foam, just as a low amount of internal interface would. Figure 3(a) shows, for $\chi N=160$, the solvent volume fraction at the center of the bubble as a function of bubble radius for various overall solvent volume fractions $\phi_{s}$. For all cases, the "quality" of the bubble is poor at the critical radii, that is, bulk conditions are not reached at the center of the bubble. In fact, all the curves fall on top of each other, indicating that for a given $\chi N$, the center volume fraction value is a universal function of radius. For $\chi N=160$, we see from this figure that a radius of about $R=0.7 R_{g}$ will give good quality bubbles. Figure 3(b) shows the corresponding cell densities at this radius for different overall solvent volume fractions. As before, the greater the dissolved gas, the higher the cell density.

Overall, we find that, consistent with experimental experience $[13,15,32$, $33,34]$, to get the "best" possible foam one should dissolve the greatest amount of blowing agent into the polymer melt while remaining away from the spinodal point, that is, the point of absolute instability of the mixture. SCFT can then be used to predict the smallest possible radius for which the interior of a typical bubble remains well segregated. If good quality bubbles are not required for an application, one may attempt to form smaller bubbles with the limit being either the maximal area to polymer volume ratio, or the critical radius itself. It is likely 


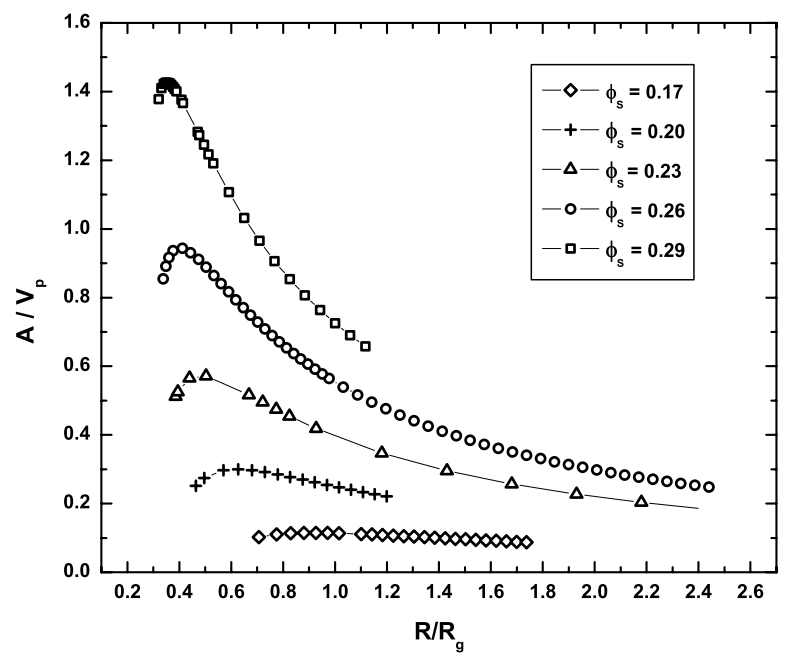

(a)

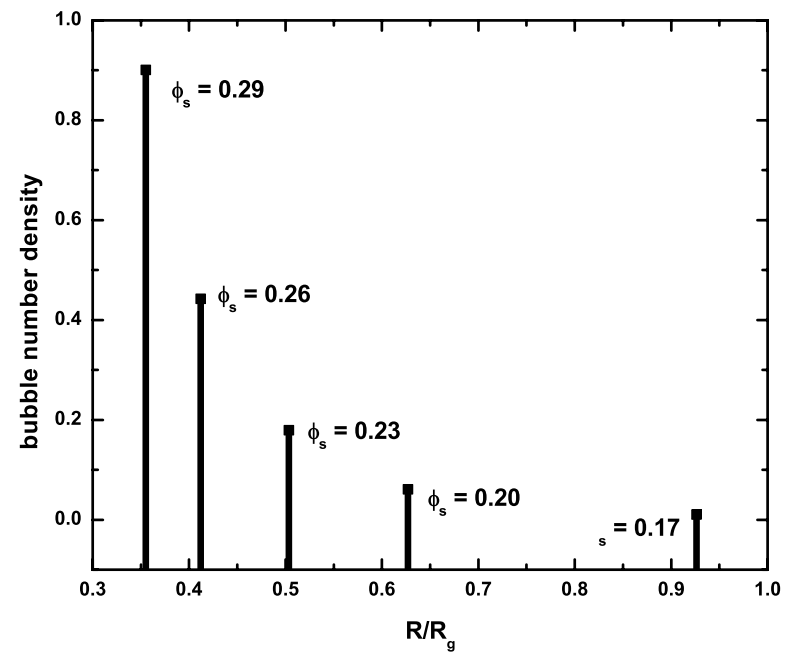

(b)

Figure 2: At $\chi N=160$. (a) Total dimensionless bubble surface area per polymer volume $V_{p}=\phi_{p} \times V$ versus bubble radius. (b) Dimensionless bubble number density at the radius of the maximum of $A / V_{P}$. 


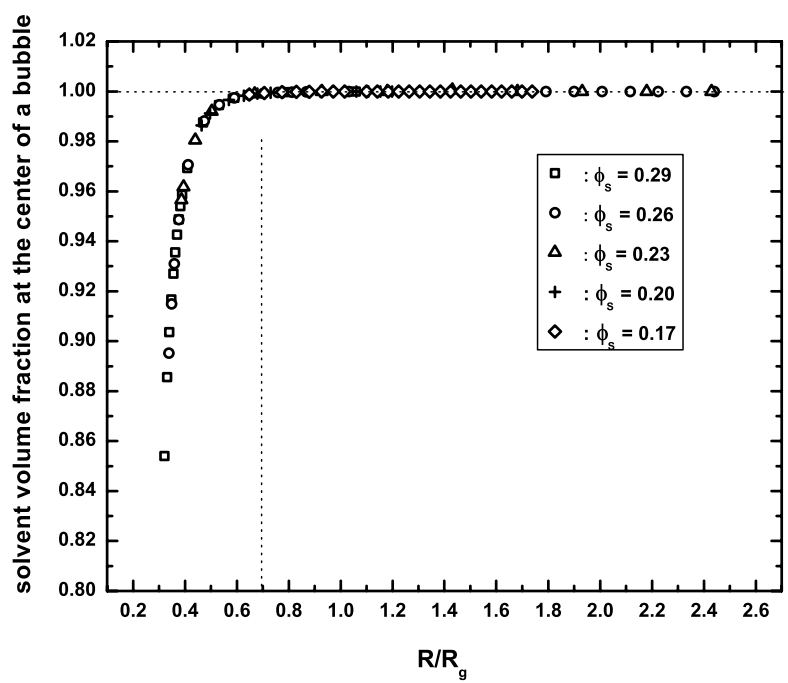

(a)

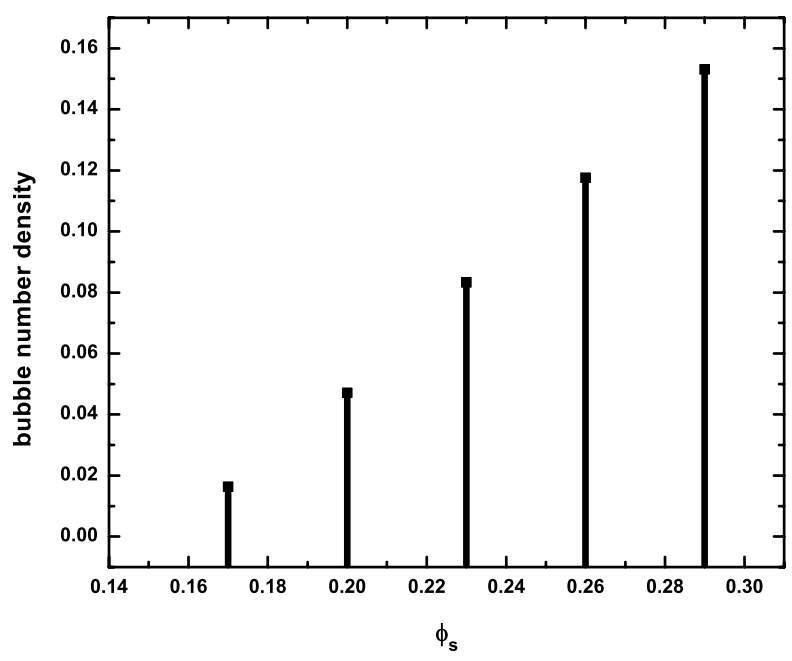

(b)

Figure 3: At $\chi N=160$. (a) Solvent volume fraction value at the center of a bubble versus radius of the bubble. (b) Dimensionless bubble number density at the bubble radius $R=0.7 R_{g}$ at different solvent density systems. 
that such small bubbles would be much more difficult to quench and therefore the bubble internal bulk condition sets an effective limit on the "smallness" of bubble achievable under given experimental conditions. SCFT can predict this condition and the accompanying maximum possible cell density. The amount of internal interface can also be easily extracted, as discussed above. Thus SCFT provides, in principle, a way of predicting the minimum possible bubble size and maximum possible cell density and interface for given conditions. Actual experiments would almost certainly fall short of this idealized limit since SCFT contains no kinetic information; real systems could often not find a kinetic pathway to the SCFT prediction. Nonetheless, such predictions could be extremely useful in excluding certain experimental conditions from consideration: SCFT can quickly survey families of conditions and identify those conditions where there is a possibility of forming a useful foam.

Since CNT, and more advanced theories based on CNT, are typically used to predict nucleation rates and through this cell densities, it is appropriate to compare SCFT to CNT. Unlike CNT, the parameters $\gamma$ and $\Delta \mathcal{F}_{V}$ are derived within the SCFT theory [16] and are found to have significant radius dependence. We calculated $\gamma$ and $\Delta \mathcal{F}_{V}$ as well as $\Delta F^{*}$ as a function of a bubble radius for a variety of segregations $\chi N$ (which is inversely proportional to temperature) and overall solvent volume fractions. At a given temperature, $\gamma$ is the same function of a bubble radius irrespective of solvent density of a system. This means that the predictions of CNT and SCFT will be qualitatively the same as far as $\Delta \mathcal{F}_{V}$ is concerned as the following example reveals. If we choose the $\chi N=160$ system with a bubble radius of $R=0.7 R_{g}$, which preserves a good quality bubble (no significant deviation from pure solvent at the center of the bubble), the surface tension is the same for different solvent density systems as shown in figure $4(\mathrm{a})$. As we increase the overall volume fraction $\phi_{s}$ however, we find, from SCFT, a larger $\Delta \mathcal{F}_{V}$ (figure $4(\mathrm{~b})$ ) and a higher bubble number density (figure $3(\mathrm{~b})$ ). From the CNT perspective, a higher $\Delta \mathcal{F}_{V}$ for constant $\gamma$ would give, from equation (4), a lower activation barrier. This lower barrier, when substituted into equation (3) gives a higher nucleation rate and, therefore, a higher cell density. Therefore both SCFT and CNT predict higher cell densities for larger gas saturation, or in other words, a bigger $\Delta \mathcal{F}_{V}$ predicts a higher cell density in both theories. Similarly, from CNT, equation (5) predicts a smaller critical bubble size with larger $\Delta \mathcal{F}_{V}$ for constant $\gamma$. Figure 3 shows this to be the case with SCFT as well.

The situation becomes more interesting when we consider a change in temperature. We examine the case of $\chi N=120$, which corresponds to a higher temperature (or different chemistry), taking volume fractions values $\phi_{s}$ ranging from 0.28 to 0.39 , within the nucleation and growth region for $\chi N=120$. Repeating the same analysis as for $\chi N=160$, we choose a fixed radius of bubble, $R=0.96 R_{g}$, for which the interior of the bubble deviates little from pure solvent for all overall volume fractions $\phi_{s}$. This is shown in figure 5. Through SCFT, we find that this increase in temperature causes the surface tension to drop, as expected, for all radii and all overall volume fractions. See figure 6 . In comparing the high and low temperature cases $(\chi N=120$ and $\chi N=160$, 


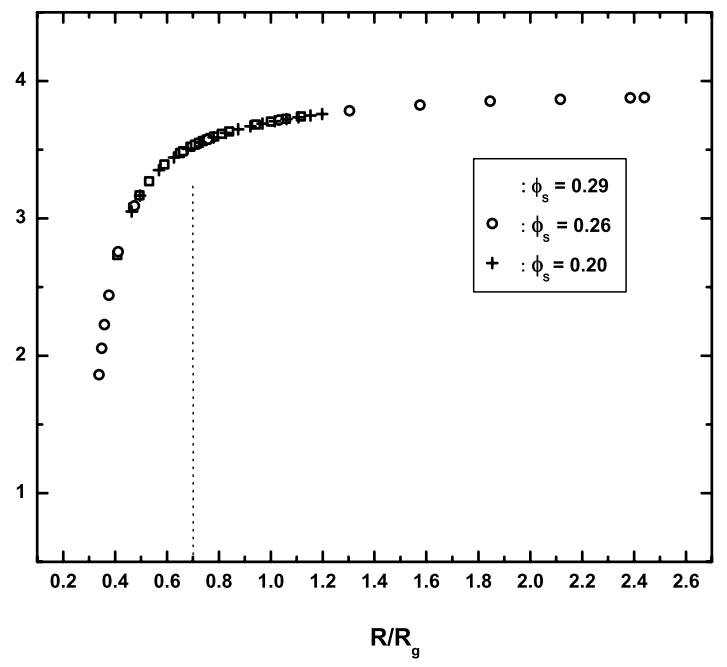

(a)

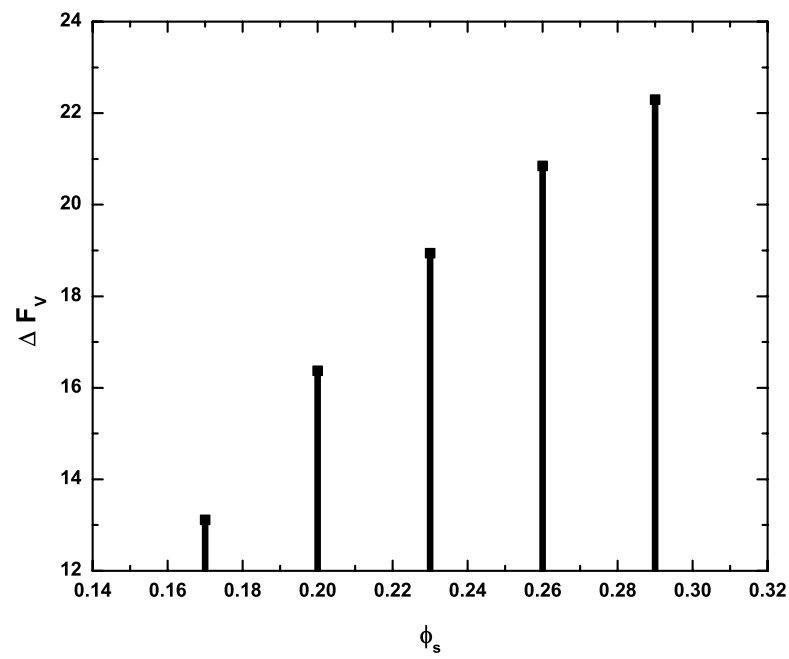

(b)

Figure 4: (a) Dimensionless surface tension $\gamma$ versus radius of a bubble at $\chi N=160$. (b) $\Delta \mathcal{F}_{V}$ at $\chi N=160$ and $R=0.7 R_{g}$. 


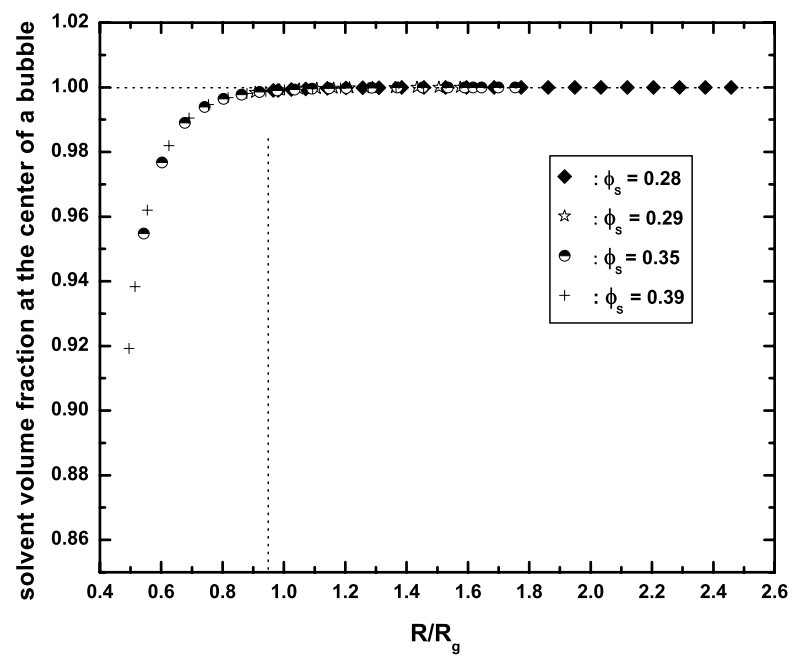

Figure 5: Solvent volume fraction at the center of a bubble versus radius of a bubble at $\chi N$ $=120$.

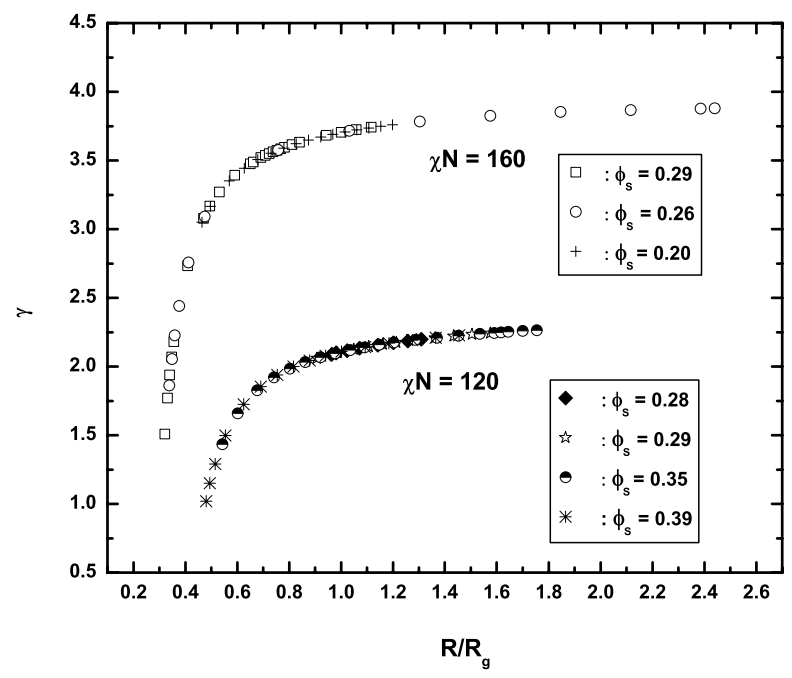

Figure 6: Dimensionless surface tension $\gamma$ at $\chi N=120$ and $\chi N=160$. 
respectively) a CNT analysis might proceed something like the following. All other factors being constant, formula (4) predicts a smaller CNT activation barrier, and therefore, from (3), a higher nucleation rate and a higher cell density for the high temperature case $(\chi N=120)$. Although SCFT predicts the intuitively expected lower surface tension for high temperature, it predicts instead a lower cell density for the high temperature, $\chi N=120$ case than for the low temperature, $\chi N=160$ case. This is shown in figure 7(a). SCFT also disagrees with the naive CNT analysis in that the critical radius is smaller for low temperatures (high surface tension) - compare equation (5). The divergence of predictions between CNT and SCFT is even more pronounced if we use the critical radii of bubbles for a given common overall solvent volume fraction rather than the preferred radius at which bulk conditions are met within the bubbles. Either way, the radius (critical or preferred) is smaller for low temperatures and the cell density is higher for low temperatures.

The cause of the disagreement can be traced to the neglect of the variation of $\Delta \mathcal{F}_{V}$ with temperature when using CNT. SCFT results, shown in figure 7 (b), reveal that $\Delta \mathcal{F}_{V}$ is smaller for higher temperatures $(\chi N=120)$, so a fair application of CNT would predict a larger critical radius, a larger activation barrier and a lower nucleation rate and ultimate cell density, in agreement with SCFT predictions. Although $\gamma$ and $\Delta \mathcal{F}_{V}$ are considered to be constants with respect to bubble size within $\mathrm{CNT}$, they are both assumed to have temperature dependence. This dependence has to be measured or calculated outside of CNT. From the above SCFT analysis, we find that $\Delta \mathcal{F}_{V}$ is by far the more important parameter in foaming processes, with $\Delta \mathcal{F}_{V}$ trends within CNT controlling the correct behaviour and $\gamma$ trends being irrelevant. For SCFT and CNT to agree, one finds that increasing the size of $\Delta \mathcal{F}_{V}$ is the best way to create a high cell density foam as opposed to trying to reduce surface tension $\gamma$. From figure 8 we see that at lower temperature (high $\chi N$ ), it is easier to achieve large $\Delta \mathcal{F}_{V}$ values even at low overall solvent volume fractions. We can therefore conclude that to make a high cell density foam, one should work at as low a temperature as possible so as to have a high $\Delta \mathcal{F}_{V}$ value, smaller bubble sizes and higher cell densities. This conclusion is in agreement with the experimental findings of Goel and Beckman [13] and many others. For some recent examples, see references $[36,15,33,37]$. The accompanying high surface tension, despite working against the above trends in principle, is subordinate to the $\Delta \mathcal{F}_{V}$ parameter. Goel and Beckman required a low surface tension at low temperatures to fit to CNT and to use CNT to account for the observed increased cell density. In fact, we see that the surface tension can and should be high at low temperatures; the cell density will increase, in contradiction to CNT. Furthermore, a non-negligible surface tension value might be expected in order to maintain the structure of the smallest foams. Wong et al., despite observing lower initial nucleation rates at low temperatures, still found marginally higher cell densities. Leung et al. also observed higher cell densities at lower temperatures and found the surface tension to have a minimal effect, as expected from SCFT. Such an "inverse" temperature dependence has been discussed in the context of non-polymeric nucleation by Talanquer et al. [38, 39]. 


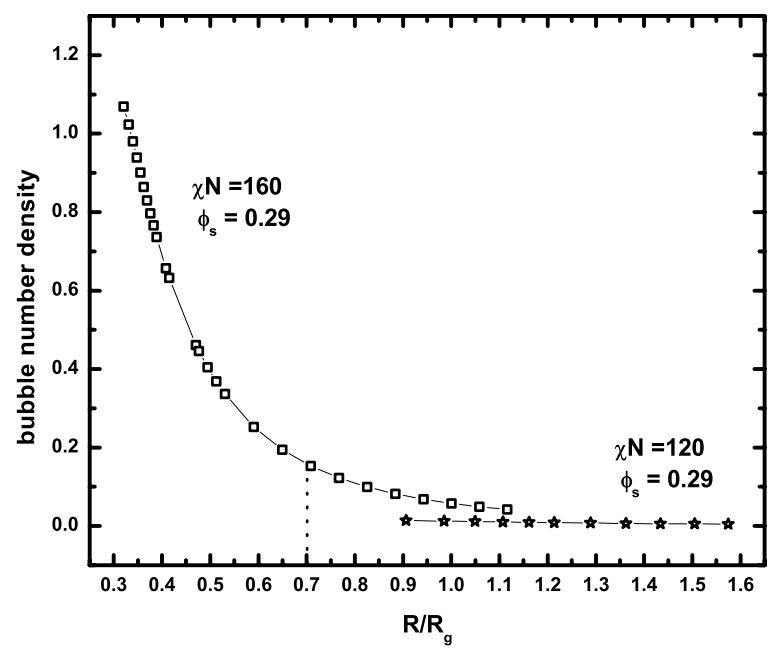

(a)

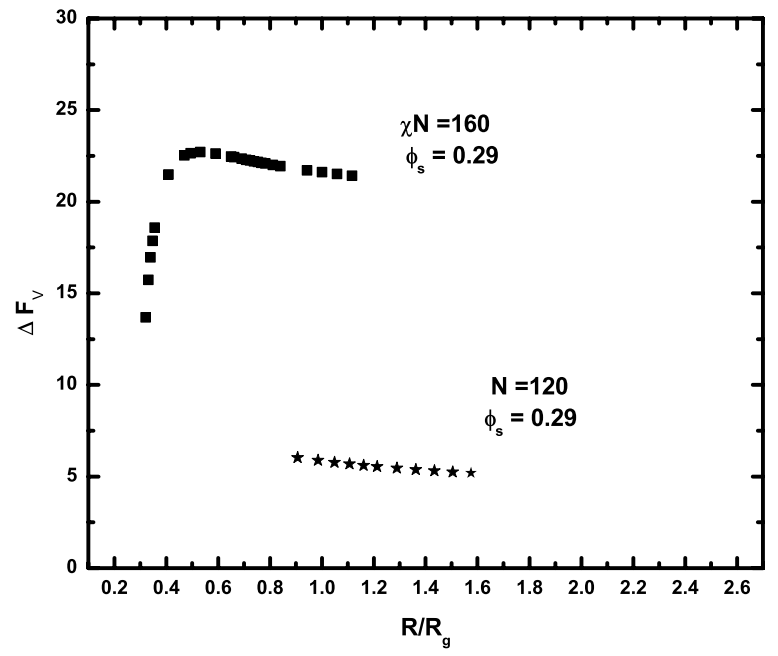

(b)

Figure 7: At $\chi N=160, \phi_{s}=0.29$ and $\chi N=120, \phi_{s}=0.29$ (a) Dimensionless bubble number density. (b) $\Delta \mathcal{F}_{V}$ versus a radius of a bubble. 


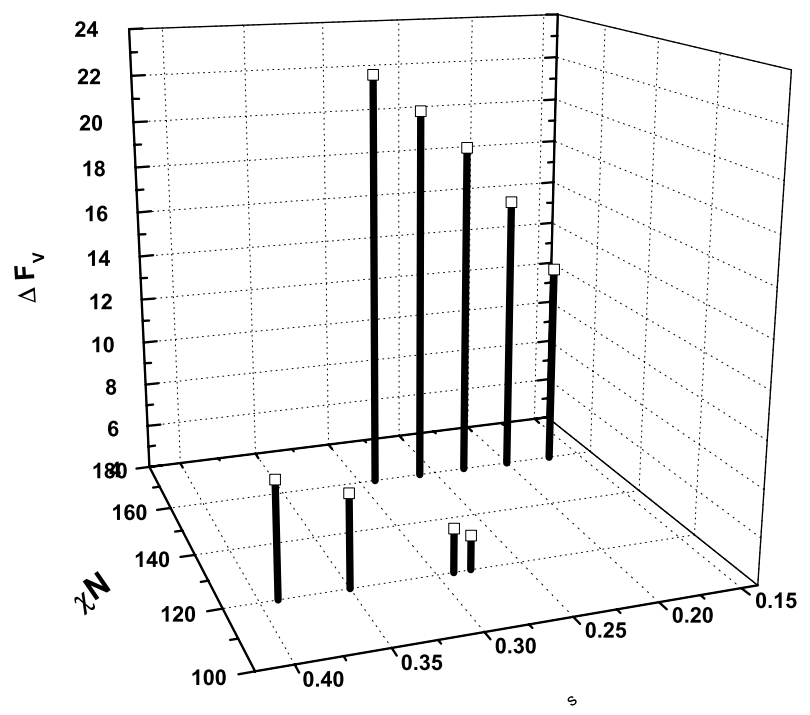

Figure 8: $\Delta \mathcal{F}_{V}$ at $\chi N=120$ and $\chi N=160$.

It may seem counter-intuitive that surface tension becomes less important for small bubbles, since the surface to volume ratio will be higher and, as a result, the surface would be expected to be increasingly important. This unexpected result can be explained in terms of the findings our of previous publication [16]. There, it was found that the surface tension drops significantly for smaller bubbles due to highly curved surfaces allowing more polymer configurations without additional energy penalties. This means that the excess free energy (free energy of the interface) drops faster than the area shrinks as we decrease the bubble size. Thus the intensive surface contribution (per unit of surface the surface tension is the excess free energy per unit area) was found to be less for small cells than for large cells. From this perspective, it's not surprising that the surface tension doesn't have a prominent role, even for small bubbles. Amon and Denson have also found the surface tension to be unimportant to the foaming process in their theoretical work [29].

\section{Conclusions}

We have demonstrated an in-principle method of determining an upper bound on the possible cell density of a polymer foam depending on conditions such as temperature, chemistry or amount of blowing agent. This self-consistent field theory method provides maximal cell densities as a function of bubble radius so that one can extract the cell density at the critical radius, the radius of 
maximum bubble surface area to polymer volume, or the radius of near complete polymer exclusion from the interior of a bubble. It is this last condition that is likely the most appropriate for many foams and it is the one that predicts the lowest cell density for any given set of experimental conditions. The method is "in-principle" in that the theory as presented assumes an incompressible equation of state. More realistic equations of state should be incorporated into the formalism for quantitative predictions. Even without such equations of state, the method reveals that when using classical nucleation theory to make predictions about polymer foam cell densities, the surface tension parameter is much less important than the volume free energy density (often phrased as a pressure difference). It is this latter parameter that dominates the behaviour of the CNT equations, at least for situations where the radius dependence of the surface tension and volume free energy density is important, such as nanocellular foams. The SCFT method provides a facile route to setting limits on the possible cell densities of polymeric foams without requiring intermediate calculations or exponential pre-factors, and is applicable for both nanocellular and more typical polymeric foams.

\section{Acknowledgements}

The authors acknowledge financial support from NSERC Canada in the form of a Strategic Projects Grant and Discovery Grants.

\section{References}

[1] S.-T. Lee, C. B. Park, N. S. Ramesh, Polymeric Foams: Science and Technology, CRC Press, Boca Raton, 2007.

[2] D. W. Oxtoby, R. Evans, Nonclassical nucleation theory for the gas-liquid transition, J. Chem. Phys. 89 (1988) 7521-7530.

[3] J. G. Lee, R. W. Flumerfelt, A refined approach to bubble nucleation and polymer foaming process: Dissolved gas and cluster size effects, J. Colloid Interf. Sci. 184 (1996) 335348.

[4] D. W. Oxtoby, Density functional methods in the statistical mechanics of materials, Annu. Rev. Mater. Res. 32 (2002) 39-52.

[5] I. J. Ford, Statistical mechanics of nucleation: a review, Proc. Instn. Mech. Engrs. Part C: J. Mechanical Engineering Science 218 (2004) 883-899.

[6] A. V. Neimark, A. Vishnyakov, Monte carlo simulation study of droplet nucleation, J. Chem. Phys. 122 (2005) 174508-1-11.

[7] J. Merikanto, E. Zapadinsky, A. Lauri, H. Vehkamäki, Origin of the failure of classical nucleation theory: Incorrect description of the smallest clusters, Phys. Rev. Lett. 98 (2007) 145702-1-4. 
[8] S. Shukla, K. W. Koelling, Classical nucleation theory applied to homogeneous bubble nucleation in the continuous microcellular foaming of the polystyrene-co2 system, Ind. Eng. Chem Res. 48 (2009) 7603-7615.

[9] I. Napari, J. Julin, H. Vehkamäki, Performance of some nucleation theories with a nonsharp droplet-vapor interface, J. Chem. Phys 133 (2010) 154503$1-7$.

[10] I. E. Parra, J. C. Gran̆a, Influence of the attractive pair-potential in density functional models of nucleation, J. Chem. Phys 132 (2010) 034702-1-9.

[11] S. Ghosh, S. K. Ghosh, Homogeneous nucleation in vapor-liquid phase transition of lennard-jones fluids: A density functional theory approach, J. Chem. Phys 134 (2011) 024502-1-8.

[12] S. K. Goel, E. J. Beckman, Generation of microcellular polymers using supercritical co2, in: Cellular Polymers 2nd International Conference, Rapra Technology Limited, Heriot-Watt University, Edinburgh, U. K., 1993, pp. 251-274.

[13] S. K. Goel, E. J. Beckman, Generation of microcellular polymeric foams using supercritical carbon dioxide 1: Effect of pressure and temperature on nucleation, Polym. Eng. Sci. 34 (1994) 1137-1147.

[14] S. Siripurapu, J. A. Coughlan, R. J. Spontak, S. A. Khan, Surfaceconstrained foaming of polymer thin films with supercritical carbon dioxide, Macromolecules 37 (2004) 9872-9879.

[15] S. N. Leung, A. Wong, Q. Guo, C. B. Park, J. H. Zong, Change in the critical nucleation radius and its impact on cell stability during polymeric foaming processes, Chem. Eng. Sci. 64 (2009) 4899-4907.

[16] Y. Kim, C. B. Park, P. Chen, R. B. Thompson, Origins of the failure of classical nucleation theory for nanocellular polymer foams, Soft Matter 7 (2011) 7351-7358.

[17] R. B. Thompson, C. B. Park, P. Chen, Reduction of polymer surface tension by crystallized polymer nanoparticles, J. Chem. Phys 133 (2010) 144913$1-7$.

[18] R. B. Thompson, J. R. MacDonald, P. Chen, Origin of change in molecularweight dependence for polymer surface tension, Phys. Rev. E 78 (2008) 030801-1-4.

[19] H. Park, R. B. Thompson, N. Lanson, C. Tzoganakis, C. B. Park, P. Chen, Effect of temperature and pressure on surface tension of polystyrene in supercritical carbon dioxide, J. Phys. Chem. B 111 (2007) 3859-3868.

[20] M. W. Matsen, Self-consistent field theory and its applications, in: G. Gompper, M. Schick (Eds.), Soft Matter Volume 1, Wiley-VCH, 2005, pp. $87-178$. 
[21] G. H. Fredrickson, The Equilibrium Theory of Inhomogeneous Polymers, Oxford University Press, New York, 2006.

[22] K. Binder, M. Müller, P. Virnau, L. G. MacDowell, Polymer + solvent systems: Phase diagrams, interface free energies, and nucleation, Adv. Polym. Sci. 173 (2005) 1-110.

[23] H. Wei, R. B. Thompson, C. B. Park, P. Chen, Surface tension of high density polyethylene (hdpe) in supercritical nitrogen: Effect of polymer crystallization, Colloids and Surfaces A: Physicochem. Eng. Aspects 354 (2010) 347-352.

[24] G. T. Dee, B. B. Sauer, The surface tension of polymer liquids, Adv. Phys. 47 (1998) 161-205.

[25] G. T. Dee, B. B. Sauer, The molecular weight and temperature dependence of polymer surface tension: Comparison of experiment with interface gradient theory, J. Colloid Interf. Sci. 152 (1992) 85-103.

[26] G. T. Dee, B. B. Sauer, Studies of polymer, copolymer, and associating liquids by melt surface tension methods and cahn-hilliard density-gradient theory, J. Colloid Interf. Sci. 162 (1994) 25-35.

[27] H. Park, C. B. Park, C. Tzoganakis, P. Chen, Effect of molecular weight on the surface tension of polystyrene melt in supercritical nitrogen, Ind. Eng. Chem. Res. 46 (2007) 3849-3851.

[28] D. G. LeGrand, G. L. Gaines, The molecular weight dependence of polymer surface tension, J. Colloid Interf. Sci. 31 (1969) 162-167.

[29] M. Amon, C. D. Denson, A study of the dynamics of foam growth: Analysis of the growth of closely spaced spherical bubbles, Polym. Eng. Sci. 24 (1984) $1026-1034$.

[30] X. Xu, C. B. Park, D. Xu, R. Pop-Iliev, Effects of die geometry on cell nucleation of ps foams blown with co2, Polym. Eng. Sci. 43 (2003) 13781390 .

[31] In our previous publication [16], we denoted dimensionless quantities with a tilde. In this paper, for simplicity, we drop this notation.

[32] S. Ito, K. Matsunaga, M. Tajima, Y. Yoshida, Generation of microcellular polyurethane with supercritical carbon dioxide, J. Appl. Polym. Sci. 106 (2007) 35813586.

[33] C. P. I. Tsivintzelis, A. G. Angelopoulou, Foaming of polymers with supercritical co2: An experimental and theoretical study, Polymer 48 (2007) $5928-5939$. 
[34] X. Han, K. W. Koelling, D. L. Tomasko, L. J. Lee, Continuous microceiiuiar poiystyrene foam extrusion with supercritical $\mathrm{Co}_{2}$, Polym. Eng. Sci. 42 (2002) 2094-2106.

[35] Spherical coordinates are used in the present calculation which forces the gas to form a spherical bubble upon phase separation. Therefore, the chosen geometry will seem compatible with nucleation and growth even when in the spinodal region. However, the energy barrier for the free energy of formation of a bubble as a function of bubble radius will disappear, indicating the spinodal point. Also, the homogeneous limit will reveal the spinodal and binodal points so that one can determine the correct range in which to work.

[36] L. M. Matuana, C. A. Diaz, Study of cell nucleation in microcellular poly(lactic acid) foamed with supercritical co2 through a continuousextrusion process, Ind. Eng. Chem. Res. 49 (2010) 2186-2193.

[37] A. Wong, S. N. Leung, G. Y. G. Li, C. B. Park, Role of processing temperature in polystyrene and polycarbonate foaming with carbon dioxide, Ind. Eng. Chem. Res. 46 (2007) 7107-7116.

[38] V. Talanquer, D. W. Oxtoby, Nucleation of bubbles in binary fluids, J. Chem. Phys. 102 (1995) 2156-2164.

[39] V. Talanquer, C. Cunningham, D. W. Oxtoby, Bubble nucleation in binary mixtures: A semiempirical approach, J. Chem. Phys. 114 (2001) 6759-6762. 